\title{
Accuracy of Electron Affinities of Atoms in Approximate Density Functional Theory
}

\author{
Donghyung Lee, * Filipp Furche, and Kieron Burke \\ Department of Chemistry, University of California, Irvine, California 92697
}

\begin{abstract}
Most approximate density functionals do not bind small atomic anions because of large self-interaction errors. Yet atomic electron affinities are often calculated using finite basis sets with surprisingly good results, despite positive highest occupied molecular orbital (HOMO) energies. We show that excellent results (better than for ionization potentials) can be obtained using standard approximate functionals evaluated on Hartree-Fock or exact-exchange densities for which the extra electron is bound. Although these good results found with limited basis sets are not accidental, we argue that this method cannot be used in general. Thus a positive HOMO indicates that the total energy should not be disregarded, only treated with caution.
\end{abstract}

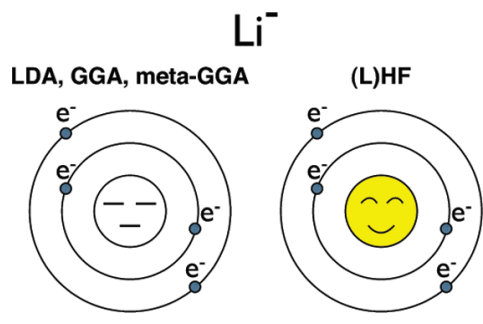

SECTION Molecular Structure, Quantum Chemistry, General Theory

$\mathrm{T}$ reating atomic anions with density functional theory (DFT) has long been controversial. ${ }^{1,2}$ The net negative charge produces strong self-interaction errors (SIES), ${ }^{3}$ resulting in such a large upward bump in the effective potential that the last electron is unbound.

In Figure 1, we show the exact Kohn-Sham (KS) potential for $\mathrm{Li}^{-}$, found from the density of a highly accurate quantum Monte Carlo (QMC) calculation with a zero-variance zero-bias estimator, ${ }^{4}$ and by inversion of the KS equations. The highest occupied molecular orbital ( $\mathrm{HOMO})$ is at $\varepsilon_{2 \mathrm{~s}}=-A$, with $A=$ $0.62 \mathrm{eV}$, the electron affinity (EA). We also show the KS potential when the exchange-correlation (XC) contribution is evaluated using the local density approximation (LDA) on this accurate density. The 2 s orbital of this potential is a very sharp resonance, at approximately $0.80 \mathrm{eV}$.

Formal theorists argue that approximate DFT does "not apply to negative atomic ions if the orbital energy (is) not negative." 5 Despite this, many have ignored these warnings, calculated EAs within DFT, and found reasonable results using reasonable basis sets. ${ }^{1}$ Because such calculations have positive HOMO energies for the anion, many authors report EAs found in this way with a note of caution. More than a decade ago, these opposing views were well expressed in refs 1 and 2, with the latter arguing for why such calculations should be discounted on formal grounds, and the former demonstrating that no practical problems arise, even with very large basis sets, and that useful results can be found for many small molecules. ${ }^{6}$

The fact remains that a formally problematic procedure yields physically meaningful results. This strongly suggests that there is a systematic property to be explored. In the present letter, we use DFT calculations with exact exchange to elucidate that structure and show how the practical and formal are reconciled. Our analysis suggests a new practical solution to the problem that is as accurate as any existing DFT method with fewer formal difficulties.
We begin with our notation and formalism. The KS equations for any atom or ion are

$$
\left[-\frac{1}{2} \nabla^{2}+v_{\mathrm{S}}^{\sigma}(r)\right] \phi_{i \sigma}(r)=\varepsilon_{i \sigma} \phi_{i \sigma}(r)
$$

where $v_{\mathrm{S}}^{\sigma}(r)$ is a single, multiplicative spin-dependent KS potential, and $\sigma$ is a spin index (up and down spins). The KS potential is written as a sum of three contributions:

$$
v_{\mathrm{S}}^{\sigma}(r)=v(r)+v_{\mathrm{H}}[n](r)+v_{\mathrm{XC}}^{\sigma}\left[n_{\uparrow}, n_{\downarrow}\right](r)
$$

where $v(r)=-Z / r$ for an atom, $v_{\mathrm{H}}(r)$ is the Hartree potential, and the $\mathrm{XC}$ potential is

$$
v_{\mathrm{XC}}^{\sigma}\left[n_{\uparrow}, n_{\downarrow}\right](r)=\frac{\delta E_{\mathrm{XC}}\left[n_{\uparrow}, n_{\downarrow}\right]}{\delta n_{\sigma}(r)}
$$

Thus, for either the exact or some approximate XC functional of the (spin) densities, we have a self-consistent set of equations.

Far from a nucleus, the Hartree potential decays as $N / r$, where $N$ is the electron number. The exact XC potential decays as

$$
v_{\mathrm{XC}}(r) \rightarrow-1 / r, \quad r \rightarrow \infty
$$

which is a pure exchange effect. ${ }^{7}$ For a neutral atom, $Z$ equals $N$, and $v_{\mathrm{S}}(r)$ approaches $-1 / r$ exactly. However, almost all local and gradient-corrected functional approximations to $v_{\mathrm{XC}}(r)$ decay incorrectly with $r$, typically exponentially, as the density decays exponentially. This has only a small effect on the density itself, but leads to very poor HOMO levels in such calculations (errors of several eV). These are all manifestations of the infamous SIE.

Received Date: May 25, 2010

Accepted Date: June 20, 2010

Published on Web Date: June 25, 2010 


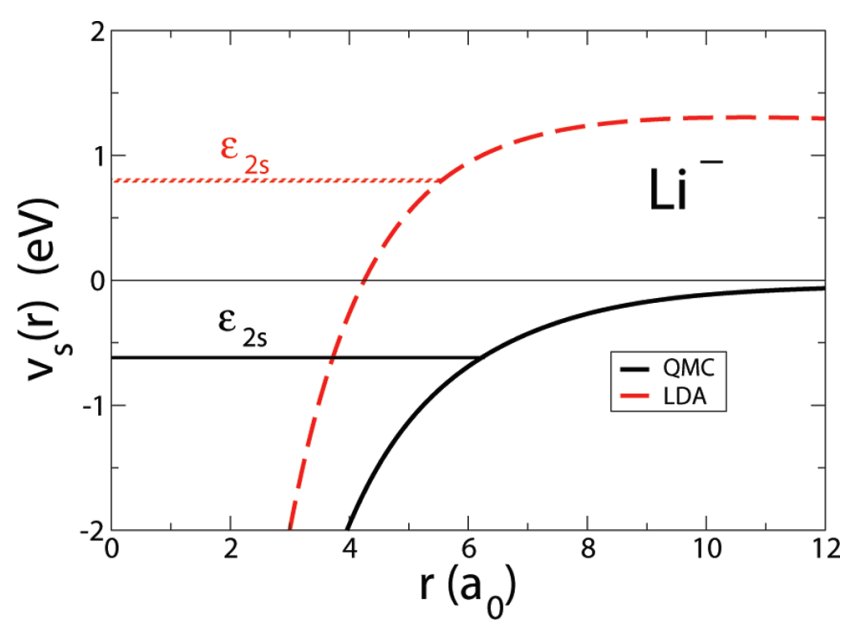

Figure 1. Comparison of KS potentials of $\mathrm{Li}^{-}$. The black line is essentially exact, using a density from OMC. The red (dashed) line is the LDA potential on that density. The horizontal lines mark the HOMO (2s orbital) energies.

A well-known cure for this problem in DFT is to use the optimized effective potential (OEP) method, ${ }^{8}$ which finds the KS potential for an orbital-dependent functional. The simplest case is to include exchange alone, often denoted EXX, producing a KS potential with the correct asymptotic behavior and a HOMO energy that is close to $-I$ for neutral systems. A practical and accurate approximation to OEP, called localized Hartree-Fock (LHF), was developed by Görling and coworkers. ${ }^{9,10}$ The total energies of EXX are practically indistinguishable from those of a standard Hartree-Fock (HF) calculation. ${ }^{11}$

While awkward and embarassing for DFT enthusiasts, this problem is not fatal for neutral atoms and cations, because one extracts the total energy by applying the energy functional to the self-consistent density, and need never look at the orbital energies. Such total energies (and especially energy differences) have been found to have chemically useful accuracy. ${ }^{12}$ However, for anions, the problem does appear to be fatal, since the exponential decay of the approximate $v_{\mathrm{XC}}(r)$ leaves a KS potential that behaves as

$$
v_{\mathrm{S}}(r) \rightarrow+1 / r, \quad r \rightarrow \infty
$$

for an anion using an approximate XC functional. This produces a large positive bump in the potential, especially for small systems, and usually raises the HOMO above the zero of the potential (value at $\infty$ ). As we show in Figure 1, the LDA HOMO energy is $0.83 \mathrm{eV}$, and the last orbital is not an eigenstate, but a very sharp resonance. The KS potential decays slowly and the classical outer turning point is at $17 \AA$. The true self-consistent ground state, using an approximate functional, is found when a sufficiently large fraction of an electron has escaped from the system (tunnelled to $\infty$ ), reducing the HOMO energy to zero. We avoid this sorry fate in Figure 1 by evaluating the LDA approximation to the KS potential on the exact density, not a self-consistent density. Note that hybrid functionals mix about $25 \%$ of exact exchange with a generalized gradient approximation (GGA). This reduces the barrier by about $25 \%$, but does not rectify the problem.
Long ago, Shore et $\mathrm{al}^{3}$ studied the LDA for the $\mathrm{H}^{-}$ground state. They confined electrons in a cavity by adding a spherical hard wall and calculated HOMO and total energies, varying the position of the wall $\left(R_{\mathrm{B}}\right)$. They found that there was a plateau-like region of the energies in 15 au $<R_{\mathrm{B}}<30 \mathrm{au}$. To obtain the asymptotic solution of $\mathrm{H}^{-}$as $R_{\mathrm{B}} \rightarrow \infty$, they varied the number of core electrons at $r<25$ au and their wave function to determine the energy minimum. The energy minimum occurred when 1.7 electrons were localized near the nucleus, 0.3 electrons were delocalized in the asymptotic tail of the density, and the HOMO eigenvalue became zero. At about the same time, Schwarz ${ }^{13}$ noted that both $\mathrm{O}^{-}$and $\mathrm{F}^{-}$ are unstable using $\mathrm{X} \alpha^{14}$ (a precursor to $\mathrm{LDA}^{15}$ ) because HOMO eigenvalues are positive.

Galbraith and Schaefer ${ }^{1}$ claim the applicability of approximate DFT for negative ions. If basis sets such as Dunning's augmented correlation consistent polarized valence double- $\zeta$ (aug-cc-pVDZ) basis set ${ }^{16,17}$ or larger are used, DFT methods with GGA and hybrid functionals can be applied to negative ions such as $\mathrm{F}^{-}$, whose outermost electron has a positive HOMO. Subsequently, Rösch and Trickey ${ }^{2}$ correctly point out that exact DFT itself has no difficulty for anions, but that the problem is with approximate functionals, as is clearly illustrated in Figure 1. They claim that there are no difficulties with the physical significance of orbital eigenvalues, positive values are permissible, and that problems can be masked by finite basis sets. Although correct, none of this necessarily implies that accurate numbers cannot be extracted from finite basis sets. Later, careful calculations by Jarecki and Davidson ${ }^{5}$ showed that, for $\mathrm{F}^{-}$, there are two plateau regions of total energy as a function of basis-set size. On the first plateau, both total energy and HOMO appear to converge, the latter to a positive value. Beyond that, the basis set probes the outside of the barrier, some density leaks out, and the HOMO falls to zero. However, the density change is so small that the total energy barely changes. Eventually, the electron completely escapes, and the HOMO falls even further.

A naive solution to this difficulty is to perform HF or the DFT version, LHF, for such systems, which produce negative HOMOs for the anions. However, for small anions, correlation effects are large, so HF total energy differences are highly inaccurate. In fact, from the total energies, many of the anions are unbound. One can also use Koopman's theorem, where I is estimated as $-\varepsilon_{\text {HOMO }}$. While an improvement, the mean absolute errors (MAEs, $0.5 \mathrm{eV}$ ) are much larger than DFT methods including correlation with a basis set. An alternative solution to the SIE problem is to perform self-interaction corrected local spin density approximation (SIC-LSDA). ${ }^{18}$ In ref 19, Cole and Perdew applied SIC-LSDA to calculate EAs for atomic systems, $Z<86$, significantly reducing errors relative to LSDA, but much less accurate than results produced here with GGAs, hybrids, and meta-GGAs.

Our suggestion is to perform calculations that include exact exchange (either LHF or HF) for self-consistent densities, but evaluate an approximate functional on that density to obtain the energy. Such a procedure has its own drawbacks, but avoids all the pitfalls mentioned above.

In Table 1, we calculate EAs using HF densities for both neutral and negative atoms. The B3LYP results were used in 
Table 1. Errors in EAs $(\mathrm{eV})^{a}$

\begin{tabular}{lcccccc}
\hline & \multirow{5}{c}{ EA } & \multicolumn{5}{c}{$\Delta E A$} \\
\cline { 5 - 7 } & exp & LDA & PBE & B3LYP & PBE0 & TPSS \\
\hline $\mathrm{H}$ & 0.75 & 0.13 & -0.11 & 0.03 & -0.17 & 0.01 \\
$\mathrm{Li}$ & 0.62 & -0.04 & -0.12 & -0.14 & -0.14 & -0.05 \\
$\mathrm{~B}$ & 0.28 & 0.36 & 0.26 & 0.04 & 0.17 & 0.12 \\
$\mathrm{C}$ & 1.26 & 0.45 & 0.23 & -0.04 & 0.12 & 0.12 \\
$\mathrm{O}$ & 1.46 & 0.41 & 0.14 & 0.01 & -0.14 & -0.09 \\
$\mathrm{~F}$ & 3.40 & 0.59 & 0.12 & -0.04 & -0.18 & -0.07 \\
$\mathrm{MAE}$ & & 0.33 & 0.16 & 0.05 & 0.15 & 0.08 \\
$\mathrm{Na}$ & 0.55 & 0.04 & -0.01 & -0.05 & -0.05 & 0.03 \\
$\mathrm{Al}$ & 0.43 & 0.17 & 0.13 & -0.08 & 0.10 & 0.06 \\
$\mathrm{Si}$ & 1.39 & 0.21 & 0.11 & -0.13 & 0.09 & 0.07 \\
$\mathrm{P}$ & 0.75 & 0.13 & 0.03 & 0.02 & -0.03 & 0.03 \\
$\mathrm{~S}$ & 2.08 & 0.27 & 0.06 & 0.01 & 0.00 & 0.01 \\
$\mathrm{Cl}$ & 3.61 & 0.36 & 0.08 & 0.01 & 0.03 & 0.02 \\
$\mathrm{MAE}$ & & 0.20 & 0.07 & 0.05 & 0.05 & 0.04 \\
total & & 0.26 & 0.12 & 0.05 & 0.10 & 0.06
\end{tabular}

${ }^{a}$ Total energies for both neutral and negative atoms are evaluated on HF-SCF densities with the aug-CC-pVDZ basis set. ${ }^{b}$ Mean absolute error.

Figure 2. Our EAs are much more accurate than the corresponding ionization potentials (typically by about a factor of 2), with mean average errors below $0.1 \mathrm{eV}$. These results change little when the basis set is expanded (either larger valence space or more diffuse functions), and our new method, using HF densities, has a well-defined basis-set limit. The traditional method of limited basis sets (LBS) can only work until the basis set probes the decay of the positive barriers of the type shown in Figure 1. Similarly good results are found for the $\mathrm{PBE}^{22}$ GGA and its hybrid, PBE0. ${ }^{23}$ The best results are with the TPSS ${ }^{24}$ meta-GGA with HF densities. The results for all functionals are better than those of ionization potentials for these elements. They also follow the usual trends for approximate functionals. The more sophisticated functionals reduce errors by a factor of $2-5$ relative to LDA.

In Figure 3, we repeat Figure 2, but now for the PBE functional. Comparison of the two shows the more systematic (and often larger) errors of the nonempirical GGA versus the empirical hybrid B3LYP. Interestingly, with our HF method for neutral and negative atoms, PBE does as well as PBEO. B3LYP and the meta-GGA, TPSS, give the best total MAE. We find almost identical results if we use LHF instead of HF densities.

Why do limited basis sets work? If the last electron is unbound in a pure DFT calculation, one can reasonably ask why limited-basis calculations yield sensible answers at all. To confirm that they do work, in Table 2, we report the results of using a limited basis set. The B3LYP and PBE numbers were used in Figure 2 and Figure 3, respectively. Without addressing the formal issues, these results are entirely sensible and very close to those of Table 1 . MAEs are almost the same (slightly worsened), and individual differences are almost all within the MAE of the given functional. How can such sensible results and excellent agreement with Table 1 come from such an apparently ill-defined procedure?

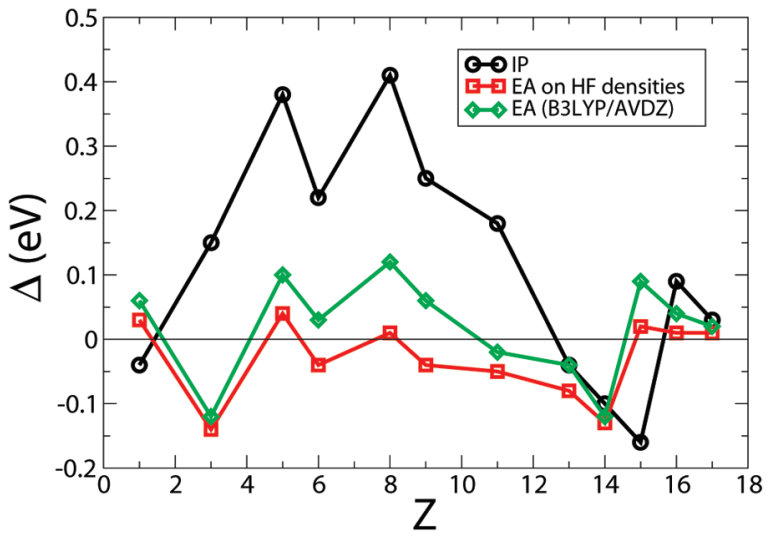

Figure 2. Comparison of errors $(\Delta)$ in ionization potentials and electron affinities in first two rows of the periodic table. Energies are evaluated with the B3LYP density functional ${ }^{20,21}$ evaluated on HF densities and on self-consistent densities within the AVDZ basis set.

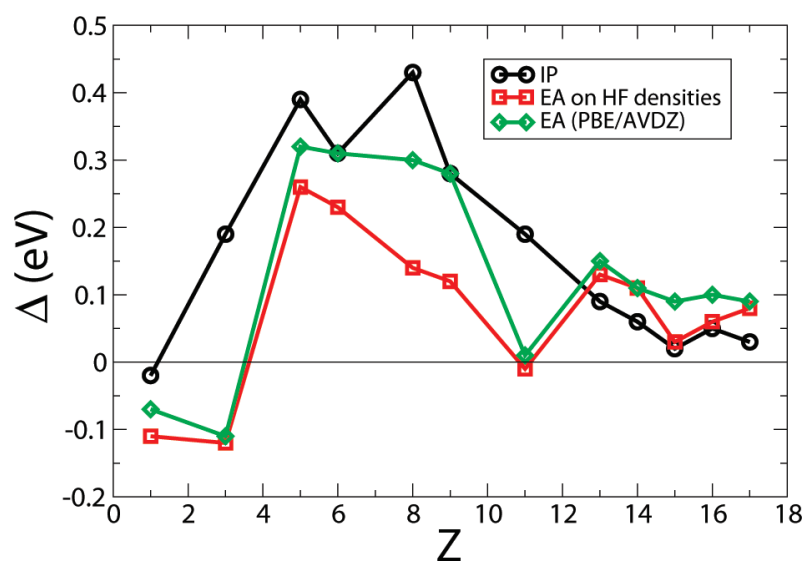

Figure 3. Comparison of errors $(\Delta)$ in ionization potentials and EAs (in eV). Energies are evaluated with the PBE density functional evaluated on the AVDZ basis set, except the square symbols, where the densities are found from an HF calculation.

The original $E_{\mathrm{XC}}$ defined by Kohn and Sham ${ }^{15}$ was for a fixed particle number. This means that the KS potential is undefined up to an arbitrary constant. The density is unaffected by an arbitrary shift of the potential. Thus positive orbital energies per se do not mean a density or total energy is inaccurate. However, in real calculations with approximate XC functionals, we conventionally set $v_{\mathrm{S}}(\rightarrow \infty)$ to zero. Thus, the electron is unbound if its orbital energy is positive, as in Figure 1. Consider a model in which we add to the potential $C \theta\left(R_{\mathrm{C}}-r\right)$, where $\theta(r)$ is the Heaviside step function and $R_{\mathrm{C}}$ is a very large fixed distance. As long as our basis sets do not stretch out to $R_{\mathrm{c}}$, the anion will appear perfectly stable and have a well-defined limit for its density. If $C$ is large enough, the HOMO will be positive. In Figure 4, we have performed just such a procedure for $\mathrm{Li}^{-}$, with $C=1.42 \mathrm{eV}$, and choosing $R_{\mathrm{C}}=$ $1000 \AA$, arbitrarily. This slightly inaccurate KS potential yields essentially the exact anionic density, produces as accurate an anionic energy as the approximate functional used to evaluate it, but has a positive HOMO of $0.80 \mathrm{eV}$. 
Table 2. Errors in EAs $(\mathrm{eV})^{a}$

\begin{tabular}{lcccccc}
\hline & \multirow{5}{c}{ EA } & \multicolumn{5}{c}{$\Delta E A$} \\
\cline { 4 - 7 } & exp & LDA & PBE & B3LYP & PBE0 & TPSS \\
\hline $\mathrm{H}$ & 0.75 & 0.15 & -0.07 & 0.06 & -0.16 & 0.02 \\
$\mathrm{Li}$ & 0.62 & -0.03 & -0.11 & -0.12 & -0.13 & -0.04 \\
$\mathrm{~B}$ & 0.28 & 0.44 & 0.32 & 0.10 & 0.19 & 0.16 \\
$\mathrm{C}$ & 1.26 & 0.53 & 0.31 & 0.03 & 0.15 & 0.18 \\
$\mathrm{O}$ & 1.46 & 0.58 & 0.30 & 0.12 & -0.07 & 0.03 \\
$\mathrm{~F}$ & 3.40 & 0.74 & 0.28 & 0.06 & -0.11 & 0.05 \\
$\mathrm{MAE}$ & & 0.41 & 0.23 & 0.08 & 0.13 & 0.08 \\
$\mathrm{Na}$ & 0.55 & 0.07 & 0.01 & -0.02 & -0.03 & 0.04 \\
$\mathrm{Al}$ & 0.43 & 0.21 & 0.15 & -0.04 & 0.11 & 0.09 \\
$\mathrm{Si}$ & 1.39 & 0.20 & 0.11 & -0.12 & 0.08 & 0.07 \\
$\mathrm{P}$ & 0.75 & 0.25 & 0.09 & 0.09 & 0.00 & 0.07 \\
$\mathrm{~S}$ & 2.08 & 0.31 & 0.10 & 0.04 & 0.01 & 0.03 \\
$\mathrm{Cl}$ & 3.61 & 0.35 & 0.09 & 0.02 & 0.02 & 0.02 \\
MAE & & 0.23 & 0.09 & 0.06 & 0.04 & 0.05 \\
total & & 0.32 & 0.16 & 0.07 & 0.09 & 0.07
\end{tabular}

${ }^{a}$ Total energies for both neutral and negative atoms are calculated by the SCF procedure with the aug-cc-pVDZ basis set.

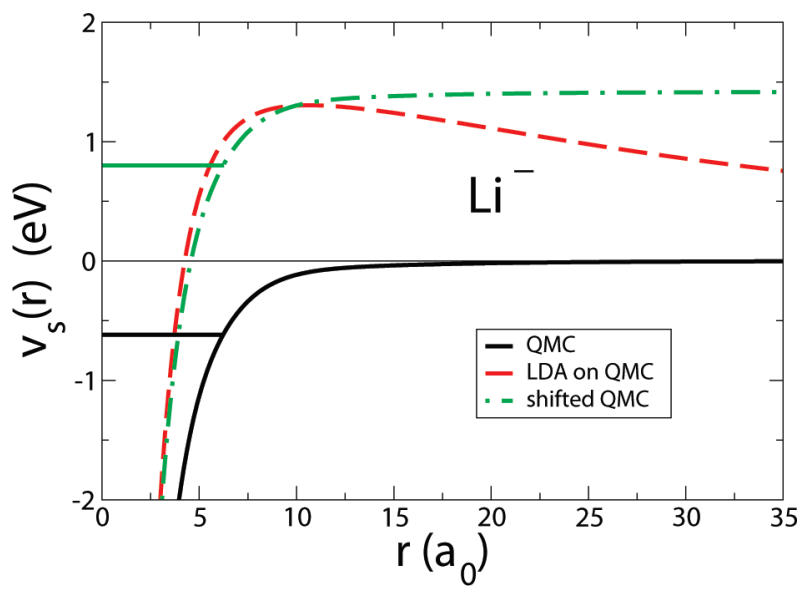

Figure 4. Shifted exact $v_{\mathrm{S}}(r)$ potential $(\mathrm{eV})$. The HOMO from QMC is $-0.62 \mathrm{eV}$, and the HOMO from LDA/AV5Z is $0.80 \mathrm{eV}$. We shift the exact $v_{\mathrm{S}}$ by the difference between eigenvalues.

Now then the question becomes, do approximate functionals, complete with SIE, really accurately mimic such a shifted KS potential? The answer has long been known to be yes, ${ }^{25}$ and we show this in Figure 5 . Here we include only the $\mathrm{XC}$ portion, in order to zoom in on the region where the exact and approximate potentials differ. The LDA potential almost exactly follows the shifted exact potential, once the outer shell (2s) is reached. Does it converge to an accurate energy and density? The answer is generally yes, if the functional is accurate except for producing the wrong asymptote.

In Figure 6, we show that the errors in density for the $\mathrm{Li}^{-}$ anion are only a few percent, and are comparable for all methods.

Finally, we investigate the dependence of HOMOs and total energies on basis sets by adding more diffuse functions for $\mathrm{F}^{-}$.

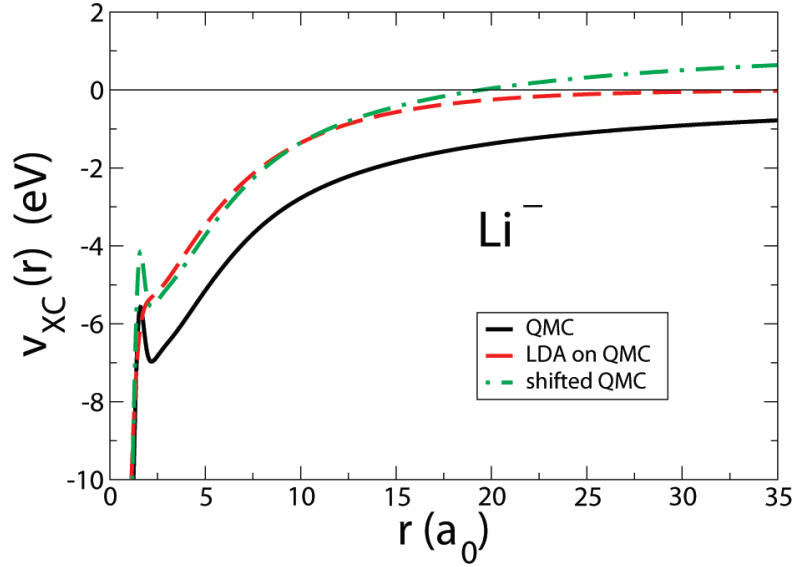

Figure 5. Shifted exact $v_{\mathrm{XC}}(r)$ potential $(\mathrm{eV})$ in $\mathrm{Li}^{-}$. The HOMO from QMC is $-0.62 \mathrm{eV}$, and the HOMO from LDA/AV5Z is $0.80 \mathrm{eV}$. We shift the exact $v_{\text {XC }}$ by the difference between eigenvalues.

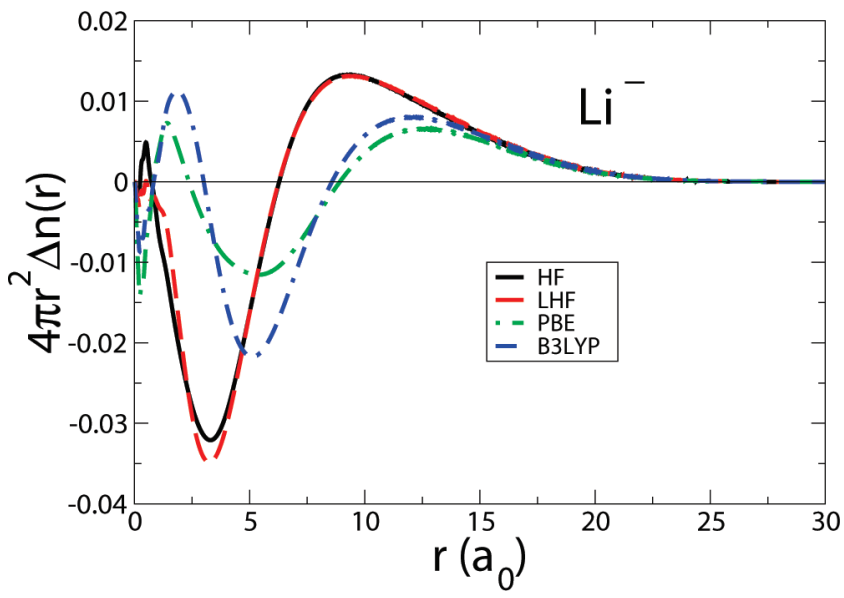

Figure 6. Plot of the radial density errors for $\mathrm{Li}^{-}$with various approximations. SCF densities are obtained with AVQZ. The exact density is from QMC.

Moving from singly augmented to quadruply augmented AVTZ basis functions, the HOMO drops from $1.57 \mathrm{eV}$ down to $0.75 \mathrm{eV}$, but the total energy of the anion changes by only $2 \mathrm{mH}$. We also checked the use of a logarithmic (diffusive) grid, but this had effects only in the $50 \mu \mathrm{H}$ regime.

In summary, we have suggested an alternative method for calculating EAs of small anions that resolves the dilemma of positive orbital energies and has a well-defined basis set limit. By evaluating the energies on LHF or HF densities, in which the last electron is properly bound with a negative HOMO, accurate and sensible results are obtained. We have also shown that the consistency and accuracy of using limited basis sets for SCF calculations with approximate functionals can be understood, despite the positive HOMO energies of the anions. But an advantage of our method is that the basis-set limit is always well-defined. Using limited basis sets could run into difficulty if the self-interaction barrier becomes too narrow or insufficiently high, making it impossible to find a plateau region. Of course, by evaluating the potential with one functional while evaluating the energy with another, various 
well-known complications arise, such as in the calculation of forces. But these difficulties are far less subtle and challenging than those of positive HOMOs.

Finally, we note that even our method will fail if the (L)HF density is insufficiently accurate or the approximate functional does not provide accurate energies. Thus we expect comparable accuracy to that found here for molecular valence anions, but the weakly bound states such as dipole- or higher multipole-bound anions ${ }^{26}$ will be much more challenging and may require self-interaction-free energy functionals along with a correct treatment of dispersion.

\section{COMPUTATIONAL DETAILS}

In all our calculations, the total energies of neutral atoms and ions are calculated using the usual self-consistent unrestricted HF, LHF, and KS-DFT. The approximate functionals in DFT calculations are LDA (S-VWN5), ${ }^{27-29} \mathrm{PBE}^{22}$ hybrid $\left(\mathrm{B} 3 \mathrm{LYP}^{20,21}\right.$ and PBEO ${ }^{23}$ ), and meta-GGA $\left(\mathrm{TPSS}^{24}\right)$ functionals. We use Dunning's augmented correlation-consistent pVXZ (aug-cc-pVXZ, X = D, T, Q, and 5; AVXZ in this paper) basis sets. ${ }^{16,17}$ For the LHF calculations for anions, we calculate the Slater potential numerically everywhere to get accurate results. The calculations with basis sets are performed with TUBOMOLE 6.2. ${ }^{30}$ For the special cases of $\mathrm{H}^{-}$and $\mathrm{Li}^{-}$, we perform fully numerical DFT calculations using an OEP code ${ }^{31}$ to calculate $v_{\mathrm{S}}(r), v_{\mathrm{XC}}(r)$, and the densities using EXX. Since this code makes a spherical approximation, we do not use it for nonspherical cases. To calculate approximate functionals on HF densities, we perform unrestricted HF calculations on both neutral and negative atoms. Then, we evaluate the total energies of atoms using HF orbitals, so the kinetic energies are those of HF.

\section{AUTHOR INFORMATION}

\section{Corresponding Author:}

*To whom correspondence should be addressed. E-mail: donghyul@ uci.edu. Phone: 949824 5742. Fax: 9498248571

ACKNOWLEDGMENT We thank Eberhard Engel for the use of his atomic OEP code, and Cyrus Umrigar and Julien Toulouse for the exact $\mathrm{Li}^{-}$data from QMC calculation. K.B. and D.L. acknowledge support under NSF CHE-0809859 and the Korea Science and Engineering Foundation Grant (No. C00063). F.F. acknowledges support under NSF CHE-0911266. D.L. thanks Dr. Henk Eshuis, Dr. Enrico Tapavicza, and John Snyder for useful discussions.

\section{REFERENCES}

(1) Galbraith, J. M.; Schaefer, H. F. Concerning the Applicability of Density Functional Methods to Atomic and Molecular Negative Ions. J. Chem. Phys. 1996, 105, 862-864

(2) Rösch, N.; Trickey, S. B. Comment on Concerning the Applicability of Density Functional Methods to Atomic and Molecular Negative Ions. J. Chem. Phys. 1997, 106, 8940-8941.

(3) Shore, H. B.; Rose, J. H.; Zaremba, E. Failure of the Local Exchange Approximation in the Evaluation of the $\mathrm{H}^{-}$Ground State. Phys. Rev. B 1977, 15, 2858-2861

(4) Toulouse, J.; Assaraf, R.; Umrigar, C. J. Zero-Variance ZeroBias Quantum Monte Carlo Estimators of the Spherically and
System-Averaged Pair Density. J. Chem. Phys. 2007, 126, $244112 / 1-11$

(5) Jarecki, A. A.; Davidson, E. R. Density Functional Theory Calculations for $\mathrm{F}^{-}$. Chem. Phys. Lett. 1999, 300, 44-52.

(6) Rienstra-Kiracofe, J. C.; Tschumper, G. S.; Schaefer, H. F.; Nandi, S.; Ellison, G. B. Atomic and Molecular Electron Affinities: Photoelectron Experiments and Theoretical Computations. Chem. Rev. 2002, 102, 231-282

(7) Perdew, J. P.; Kurth, S. Density Functionals for Non-relativistic Coulomb Systems in the New Century. In Primer in Density Functional Theory (Lecture Notes in Physics); Fiolhais, C., Nogueira, F., Marques, M. A. L., Eds.; Springer-Verlag: Berlin, Germany, 2003; Vol. 620, pp 1-55.

(8) Talman, J. D.; Shadwick, W. F. Optimized Effective Atomic Central Potential. Phys. Rev. A 1976, 14, 36-40.

(9) Della Sala, F; Görling, A. Efficient Localized Hartree-Fock Methods as Effective Exact-Exchange Kohn-Sham Methods for Molecules. J. Chem. Phys. 2001, 115, 5718-5732.

(10) Weimer, M.; Della Sala, F.; Görling, A. The Kohn-Sham Treatment of Anions via the Localized Hartree-Fock Method. Chem. Phys. Lett. 2003, 372, 538-547.

(11) Görling, A.; Ernzerhof, M. Energy Differences between Kohn-Sham and Hartree-Fock Wave Functions Yielding the Same Electron Density. Phys. Rev. A 1995, 51, 4501-4513.

(12) Rappoport, D.; Crawford, N. R. M.; Furche, F.; Burke, K. Approximate Density Functionals: Which Should I Choose? In Computational Inorganic and Bioinorganic Chemistry; Solomon, E. I., Scott, R. A., King, R. B., Eds.; Wiley: Chichester, U.K., 2009; pp 159-172.

(13) Schwarz, K. Instability of Stable Negative Ions in the $\mathrm{X} \alpha$ Method or Other Local Density Functional Schemes. Chem. Phys. Lett. 1978, 57, 605-607.

(14) Slater, J. C. Quantum Theory of Molecules and Solids; McGraw-Hill: New York, 1974; Vol. 4.

(15) Kohn, W.; Sham, L. J. Self-Consistent Equations Including Exchange and Correlation Effects. Phys. Rev. 1965, 140, A1133-A1138.

(16) Dunning, T. H. Gaussian Basis Sets for Use in Correlated Molecular Calculations. I. The Atoms Boron through Neon and Hydrogen. J. Chem. Phys. 1989, 90, 1007-1023.

(17) Woon, D. E.; Dunning, T. H. Gaussian Basis Sets for Use in Correlated Molecular Calculations. III. The Atoms Aluminum through Argon. J. Chem. Phys. 1993, 98, 1358-1371

(18) Perdew, J. P.; Zunger, A. Self-Interaction Correction to DensityFunctional Approximations for Many-Electron Systems. Phys. Rev. B 1981, 23, 5048-5079.

(19) Cole, L. A.; Perdew, J. P. Calculated Electron Affinities of the Elements. Phys. Rev. A 1982, 25, 1265-1271.

(20) Becke, A. D. Density-Functional Thermochemistry. III. The Role of Exact Exchange. J. Chem. Phys. 1993, 98, $5648-5652$

(21) Lee, C.; Yang, W.; Parr, R. G. Development of the ColleSalvetti Correlation-Energy Formula into a Functional of the Electron Density. Phys. Rev. B 1988, 37, 785-9.

(22) Perdew, J. P.; Burke, K.; Ernzerhof, M. Generalized Gradient Approximation Made Simple. Phys. Rev. Lett. 1996, 77, 38653868.

(23) Perdew, J. P.; Emzerhof, M.; Burke, K. Rationale for Mixing Exact Exchange with Density Functional Approximations. J. Chem. Phys. 1996, 105, 9982-9985.

(24) Tao, J.; Perdew, J. P.; Staroverov, V. N.; Scuseria, G. E. Climbing the Density Functional Ladder: Nonempirical Meta-Generalized Gradient Approximation Designed for Molecules and Solids. Phys. Rev. Lett. 2003, 91, 146401 
(25) Perdew, J. P. What Do the Kohn-Sham Orbitals Mean? How Do Atoms Dissociate? In Density Functional Methods in Physics; Dreizler, R. M., da Providencia, J., Eds.; Plenum: New York, 1985; p 265.

(26) Simons, J. Molecular Anions. J. Phys. Chem. A 2008, 112, 6401-6511.

(27) Dirac, P. A. M. Quantum Mechanics of Many-Electron Systems. Proc. R. Soc. London, Ser. A 1929, 123, 714-733.

(28) Slater, J. C. A Simplification of the Hartree-Fock Method. Phys. Rev. 1951, 81, 385-390.

(29) Vosko, S. H.; Wilk, L.; Nusair, M. Accurate Spin-Dependent Electron Liquid Correlation Energies for Local Spin Density Calculations: A Critical Analysis. Can.J. Phys. 1980, 58, $1200-$ 1211.

(30) TURBOMOLE V6.2; TURBOMOLE GmbH: Karlsruhe, Germany, 2010; http://www.turbomole.com.

(31) Engel, E. OPMKS: Atomic DFT Program; University of Frankfurt: Frankfurt, Germany, 1997. 